\title{
A giant groin lipoma mimicking an inguinal hernia: A case report
}

\author{
Mehmet Eren Yuksel', Funda Tamer ${ }^{2}$, Esra $\mathrm{Oz}^{3}$
}

\author{
${ }^{1}$ Aksaray University School of Medicine, Department of General Surgery, Aksaray, Turkey, ${ }^{2}$ Ufuk University School of \\ Medicine, Department of Dermatology, Ankara, Turkey ${ }^{3}$ Aksaray University Training and Research Hospital, Department of \\ Pathology, Aksaray, Turkey
}

Corresponding author: Dr. Funda Tamer, E-mail: fundatmr@yahoo.com

\begin{abstract}
An inguinal hernia repair operation is a common procedure in general surgery. However, several other conditions such as inguinal lymphadenopathy, groin lipoma, spermatic-cord lipoma and sarcoma may have similar clinical findings with inguinal hernia. Preoperative diagnosis is crucial to make the appropriate treatment plan. An accurate preoperative diagnosis prevents both unnecessary operations and surgical complications. Radiological imaging techniques such as ultrasonography, computed tomography and magnetic resonance imaging should be considered in the differential diagnosis of inguinal masses. Hereby, we present a 4l-year-old Caucasian male patient with a giant lipoma in the right groin which mimicked an inguinal hernia. Preoperative ultrasound imaging helped us to make an initial diagnosis of lipoma which was subsequently confirmed by histopathological examination.
\end{abstract}

Key words: Hernia; Inguinal; Lipoma

\section{INTRODUCTION}

Lipomas are benign adipose tumors which usually present in the subcutaneous tissues. Lipomas are characterized as slow-growing, soft, mobile, round and asymptomatic masses on the head, neck, shoulders, and the back. They usually develop between the ages of 40-60. Solitary lipomas are more common in women, whereas multiple lesions usually appear in men. Histopathologically, lipomas consist of mature adipocytes with a fibrous capsule. The differential diagnosis includes epidermoid cyst, subcutaneous tumors, nodular fasciitis, liposarcoma, metastatic disease, erythema nodosum, nodular subcutaneous fat necrosis, Weber-Christian panniculitis, vasculitic nodules, rheumatic nodules, sarcoidosis and hematoma. Since lipomas are generally not painful, they usually do not require any further treatment. Even it is rare, malignancy should be kept in mind especially in fast-growing tumors. Magnetic resonance imaging (MRI) may be useful to differentiate lipomas from liposarcomas. Steroid injections, liposuction, and surgical excision are the possible treatment options [1].

\section{CASE REPORT}

A 4l-year-old Caucasian male patient with an asymptomatic inguinal swelling presented for further clinical evaluation. The patient stated that the lesion gradually enlarged within the past 5 years. The patient confirmed that he did not receive any prior treatment. Past medical history and family history were both unremarkable. The physical examination revealed a skin-colored, soft, well-defined, subcutaneous mass in the right inguinal region (Figs. 1 and 2). Ultrasonography showed an isoechoic, subcutaneous nodule with regular borders measuring 9x6x4 cm in size. Laboratory tests including complete blood count, C-reactive protein, INR and urinalysis were all in normal limits. Serum levels of hepatitis B surface antigen, antibodies against hepatitis $\mathrm{C}$ virus and anti-human immunodeficiency virus antibody were all negative. The biochemistry 
panel was normal except the slightly increased serum glucose; $115 \mathrm{mg} / \mathrm{dL}$ (normal range: $70-110 \mathrm{mg} / \mathrm{dL}$ ) and alanine aminotransferase; $43 \mathrm{u} / \mathrm{L}$ (normal range:

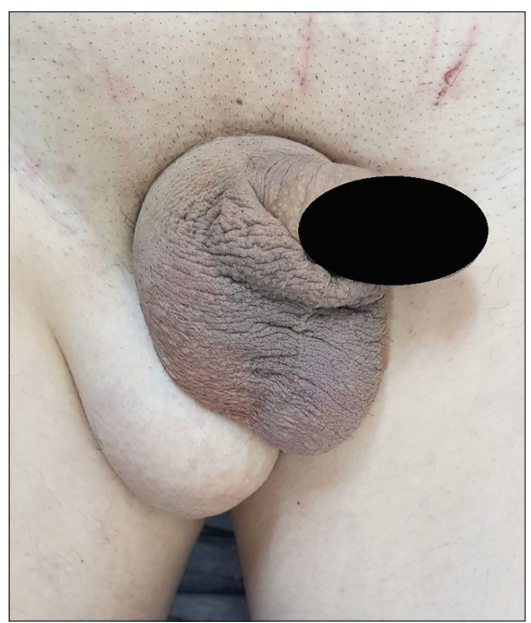

Figure 1: A skin-colored, well-defined, subcutaneous mass in the right groin measuring $9 \times 6 \times 4 \mathrm{~cm}$ in size

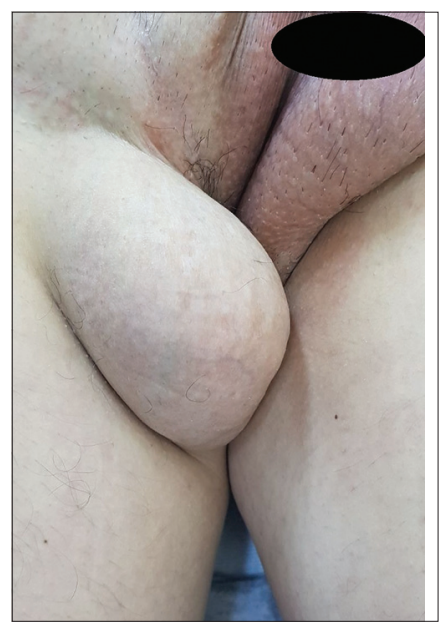

Figure 2: Perioperative view of the lesion

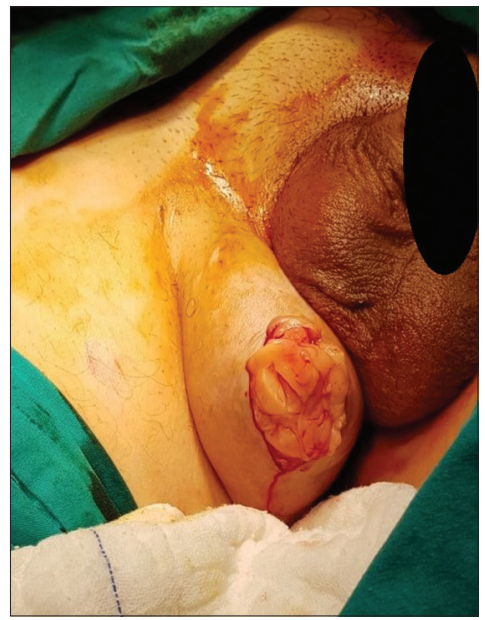

Figure 3: Intraoperative view of the lesion
0-40 $\mathrm{u} / \mathrm{L})$. The lesion in the right inguinal region of the patient was completely removed under local anesthesia with $2 \%$ prilocaine (Citanest ${ }^{\circledR}$ ) (Figs. 3 and 4$)$. The histopathological examination of the lesion revealed a giant lipoma with mature adipocytes surrounded by a fibrous capsule (Figs. 5 and 6).

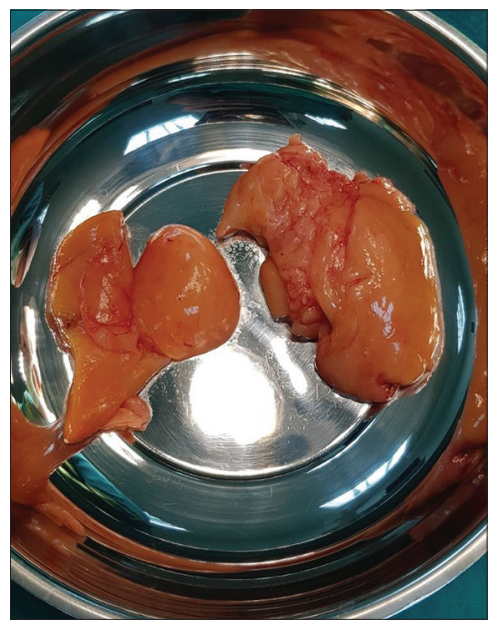

Figure 4: Surgically excised specimen

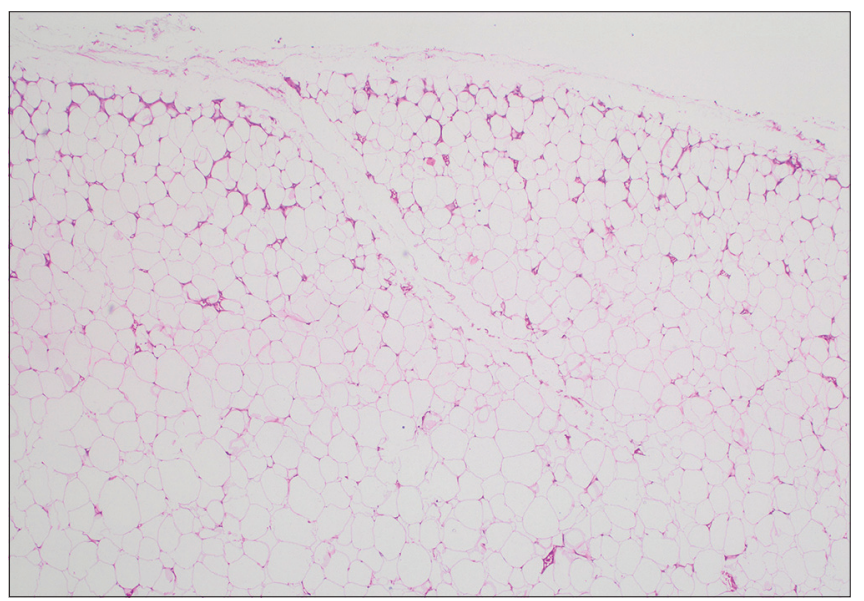

Figure 5: Mature adipocytes separated by a fibrous septa (H\&Ex40)

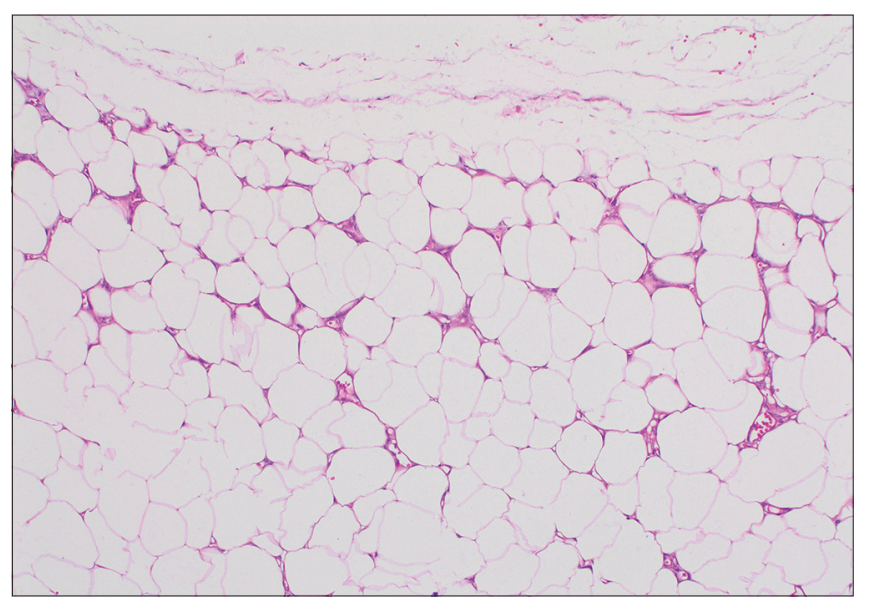

Figure 6: Closer appearance of the adipocytes (H\&Ex100) 
Prior to the study, patient gave written consent to the examination and biopsy after having been informed about the procedure.

\section{DISCUSSION}

A lipoma in the inguinal region can easily be misdiagnosed as an inguinal hernia, since both a lipoma and an inguinal hernia may lead to similar symptoms and physical examination findings. Gerych et al. reported a 70-year-old female patient with a $20 \mathrm{x} 12 \mathrm{~cm}$ sized lipoma in the right groin mimicking an inguinal hernia. Gerych et al. stated that they performed preoperative ultrasonography and computed tomography (CT) in order to differentiate a lipoma from an inguinal hernia, aneurysm of great saphenous vein, inguinal lymphadenopathy and a cold abscess [2] .

Another clinical entity which presents with inguinal hernia signs and symptoms is a pure spermatic-cord lipoma. Jo et al. reported a 33-year-old male patient with a spermatic-cord lipoma which was initially misdiagnosed as an inguinal hernia. Spermatic-cord lipomas are usually detected incidentally during the hernia repair surgery. Therefore, Jo et al. advised to perform an abdominopelvic CT and MRI in patients with a slowly growing, non-reducible, fixed, relatively hard and non-tender mass [3]. Moreover, an encysted spermatic cord hydrocele was described in a male patient as a painful, well circumscribed, $4 \mathrm{~cm}$ sized swelling in the left inguinal region which clinically mimicked an incarcerated inguinal hernia [4]. In addition, a sarcoma is a rare, malignant, soft tissue tumor which usually occurs in the thigh and retroperitoneum. A sarcoma should also be kept in mind in the differential diagnosis of an inguinal hernia. Therefore, preliminary diagnosis of a lump in the inguinal region is crucial both to offer the best available treatment option and to perform the most appropriate surgery [5].

In conclusion, inguinal hernia repair is a common surgical procedure in general surgery. However, several other conditions such as a giant lipoma can clinically mimick an inguinal hernia. In such cases, radiological examinations such as ultrasonography, CT and MRI may be helpful to reach a preliminary diagnosis prior to the surgical intervention.

\section{CONSENT}

The examination of the patient was conducted according to the Declaration of Helsinki principles.

\section{REFERENCES}

1. Salam GA. Lipoma excision. Am Fam Physician. 2002;65:901-4.

2. Gerych I, Ivankiv T, Ogurtsov O, Kalynovych N. Giant right groin lipoma mimicking inguinal hernia. Int J Surg Case Rep. 2015;12:106- 7.

3. Jo DI, Choi SK, Kim SH, Kim CK, Chung H, Kim HS. The case of huge pure lipoma of the spermatic cord misdiagnosed as inguinal hernia. Urol Case Rep. 2017;13:10-2.

4. Manimaran D, Karthikeyan TM, Khan DM. Encysted spermatic cord hydrocele in a 60-year-old, mimicking incarcerated inguinal hernia: A case report. J Clin Diagn Res. 2014;8:153-4.

5. Valeshabad AK, Walsh A, Lloyd GL. An important mimic of inguinal hernia. Urology. 2016;97:e11.

Copyright by Mehmet Eren Yuksel, et al. This is an open-access article distributed under the terms of the Creative Commons Attribution License, which permits unrestricted use, distribution, and reproduction in any medium, provided the original author and source are credited.

Source of Support: Nil, Conflict of Interest: None declared. 